\title{
ON THE COEFFICIENTS OF CERTAIN MODULAR FORMS BELONGING TO SUBGROUPS OF THE MODULAR GROUP*
}

BY

HERBERT S. ZUCKERMAN†

1. Introducton. The Fourier coefficients of modular forms $F(\tau)$ of positive dimension have been determined $\ddagger$ for the case in which $F(\tau)$ belongs to the full modular group. It is the purpose of this paper to generalize some of those results to the case where $F(\tau)$ belongs to an arbitrary subgroup of the modular group subject to certain restrictions.

By $F(\tau)$ belonging to a subgroup $\gamma$ we mean that $F(\tau)$ is analytic in the upper half-plane $I(\tau)>0$ and that $F(\tau)$ satisfies a transformation equation

$$
F\left(\tau^{\prime}\right)=\epsilon(-i(c \tau+d))^{-\tau} F(\tau)
$$

for every transformation

$$
\tau^{\prime}=\frac{a \tau+b}{c \tau+d}
$$

of $\gamma$. In (1.11) $r$, which we shall assume throughout to be positive, is the dimension of $F(\tau)$ and $\epsilon=\epsilon(a, b, c, d)$ is of absolute value one and depends only on the transformation (1.12). If $c \neq 0$ we take $c>0$, assign

$$
-\frac{\pi}{2}<\arg (-i(c \tau+d))<\frac{\pi}{2},
$$

and define $(-i(c \tau+d))^{-r}$ as $|c \tau+d|^{-r} \exp \{-i r \arg (-i(c \tau+d))\}$.

In the case of the full modular group, a Fourier expansion for $F(\tau)$ was found by considering a parabolic transformation which had infinity as fixed point. In the case of a subgroup $\gamma$ we must consider a set of parabolic transformations such that no two of the fixed points of the transformations are equivalent under $\gamma$. For these fixed points we take parabolic vertices of a fundamental region of $\gamma$. The expansions corresponding to the point at infinity are simpler than those corresponding to finite points. However, in general, the fundamental region will have more than one parabolic point; so

* Presented to the Society, February 26, 1938; received by the editors February 17, 1938.

† National Research Fellow.

$\ddagger$ H. Rademacher and H. S. Zuckerman, On the Fourier coefficients of certain modular forms of positive dimension, Annals of Mathematics, (2), vol. 39 (1938), pp. 433-462. 
some finite points will have to be considered. In order to be able to treat all the expansions symmetrically, we choose a fundamental region which does not have the point at infinity as a vertex. We then show, in $\S 3$, that $F(\tau)$ has a set of expansions of the form

$$
\begin{aligned}
& F(\tau)=\left(-i\left(\tau-P_{o}\right)\right)^{r} x^{\alpha_{\theta}} \sum_{m} a_{m}^{(g)} x^{m}=\left(-i\left(\tau-P_{o}\right)\right)^{r} x^{\alpha_{0}} f_{o}(x), \\
& x=\exp \left\{-2 \pi i / c_{\theta}\left(\tau-P_{\vartheta}\right)\right\}, \quad g=1,2, \cdots, s,
\end{aligned}
$$

corresponding to the parabolic points $P_{0}$.

In order that $s$ in (1.2) be finite we assume that $\gamma$ is of finite index in the full modular group. This is the only restriction that we place on $\gamma$. We make use of the fact that the subgroup is defined by its fundamental region and hence do not need any arithmetical characterization of it. That is, the existence in $\gamma$ of all the transformations which we use is an immediate consequence of the form of the fundamental region.

To the conditions that $r$ is positive and that $F(\tau)$ is analytic for $I(\tau)>0$ we add the restriction that $F(\tau)$ shall have only polar singularities, measured in the uniformizing variable $x$ of (1.2), at the points $P_{\boldsymbol{g}}$. That is, we assume that only a finite number of $a_{m}{ }^{(0)}$ with negative $m$ are different from zero. In $\$ \$ 5$ and 6 we find expressions for the $a_{m}{ }^{(0)}$ for $m>0$ in terms of the $a_{m}{ }^{(0)}$ for $m<0$.

The expansions (1.2) which we obtain are Fourier expansions in the variable $\left(\tau-P_{o}\right)^{-1}$. These expansions can be transformed into usual Fourier expansions in the variable $\tau$. We consider any transformation of the full modular group

$$
\tau^{\prime}=\frac{A \tau+B}{C \tau+D}
$$

and write

$$
F\left(\tau^{\prime}\right)=\epsilon^{*}(-i(C \tau+D))^{-r} F^{*}(\tau)
$$

where $F^{*}(\tau)$ depends on the choice of the transformation (1.31) as well as on $\tau$. In $\S 3$ we find an expression, (3.23), for $F^{*}(\tau)$ in terms of the $f_{\sigma}(x)$ which is, in fact, a usual Fourier expansion in $\tau$ since, by definition, we have

$$
f_{o}(x)=\sum_{m} a_{m}^{(o)} x^{m}
$$

In particular, we can obtain a usual Fourier expansion for $F(\tau)$ itself by choosing the identity transformation for (1.31). 
In $\$ 7$ we specialize the results of $\$ \S 5$ and 6 to the case of a particular subgroup, evaluating all the constants which depend only on the choice of $\gamma$. In $\S 8$ we consider the function $\vartheta_{2}(0 \mid \tau)^{-1}$ which belongs to the subgroup of $\S 7$. From the $F^{*}(\tau)$ of $(1.32)$ we then get expansions for $\vartheta_{2}(0 \mid \tau)^{-1}, \vartheta_{3}(0 \mid \tau)^{-1}$, and $\vartheta_{4}(0 \mid \tau)^{-1}$. This particular function is considered because it was partially treated by Hardy and Ramanujan. $\dagger$ They considered only the expansion of $\vartheta_{4}(0 \mid \tau)^{-1}$ and obtained results which compare with ours in the same way as their results for the partition function compare with those obtained by Rademacher. $\ddagger$

2. The fundamental region. We choose a fundamental region $R$ of $\gamma$ which we shall keep fixed throughout the discussion. Although we could use any fundamental region, we find it convenient, for symmetry, to choose one that does not have the point at infinity as a vertex. This choice is clearly possible since the subgroup $\gamma$ is of finite index. Such a region $R$ can be obtained from a fundamental region $R^{\prime}$ of $\gamma$, which has the point at infinity as a vertex, by subjecting $R^{\prime}$ to a transformation of $\gamma$ which takes the point at infinity into a finite point and which does not take any of the finite vertices of $R^{\prime}$ into infinity.

Under $\gamma$ every real rational point transforms into a real rational point or infinity. Since every real rational point is equivalent to a point of $R$, we see that each is equivalent to a real rational point of $R$. Also every real point of $R$ is a parabolic vertex; so we see that every real rational point is equivalent under $\gamma$ to a parabolic vertex of $R$.

Some of the parabolic vertices of $R$ may be equivalent under $\gamma$ thus forming cycles of more than one vertex. We pick out a representative vertex from each cycle and name the vertices $P_{1}, P_{2}, \cdots, P_{s}$. Since the $P_{g}$ are real rational points, we can write

$$
P_{o}=\frac{p_{o}}{q_{o}}, \quad\left(p_{o}, q_{o}\right)=1, \quad q_{o}>0, \quad g=1,2, \cdots, s .
$$

3. The functions $f_{o}(x)$. Corresponding to each parabolic vertex $P_{o}$ there is a transformation of $\gamma$ which may be written

$$
\frac{1}{\tau^{\prime}-P_{g}}=\frac{1}{\tau-P_{o}}+c_{o}, \quad c_{o}>0
$$

Putting this transformation in our standard form, we have

$\dagger$ Asymptotic formulae in combinatorial analysis, Proceedings of the London Mathematical Society, (2), vol. 17 (1918), pp. 75-115; also S. Ramanujan, Collected Papers, pp. 276-309.

$\ddagger$ On the partition function $p(n)$, Proceedings of the London Mathematical Society, (2), vol. 43 (1937), pp. 241-254. 


$$
\tau^{\prime}=\frac{\delta^{-1}\left(q_{o} c_{o} p_{o}+q_{\theta}^{2}\right) \tau-\delta^{-1} c_{\sigma} p_{\theta}^{2}}{\delta^{-1} q_{o}^{2} c_{o} \tau+\delta^{-1}\left(q_{o}^{2}-c_{o} p_{o} q_{\theta}\right)}
$$

where $\delta=\left(q_{\theta} c_{\theta} p_{\theta}+q_{\theta}^{2}, c_{\theta} p_{\theta}^{2}, q_{\theta}^{2} c_{\theta}, q_{\theta}^{2}-c_{\theta} p_{\theta} q_{\theta}\right)=\left(q_{\theta}^{2}, c_{\theta}\right)$. However this is a modular transformation; so its determinant is unity. Hence we have

$$
q_{\theta}^{4}-c_{0}^{2} p_{\theta}^{2} q_{\theta}^{2}+c_{\theta}^{2} p_{\theta}^{2} q_{\theta}^{2}=\delta^{2}, \quad \delta= \pm q_{\theta}^{2} ;
$$

so $q_{\sigma}^{2}$ divides $c_{g}$ and we may write the transformation in our standard form as

$$
\tau^{\prime}=\frac{\left(c_{\theta} P_{0}+1\right) \tau-c_{\theta} P_{\theta}^{2}}{c_{\theta} \tau+1-c_{\theta} P_{\theta}}
$$

Then, by (1.11), we have

$$
F\left(\tau^{\prime}\right)=\epsilon\left(-i\left(c_{g} \tau+1-c_{g} P_{g}\right)\right)^{-r} F(\tau),
$$

or, writing $T=\left(\tau-P_{\vartheta}\right)^{-1}, T^{\prime}=\left(\tau^{\prime}-P_{\vartheta}\right)^{-1}=T+c_{0}$,

$$
\begin{aligned}
F\left(\frac{1}{T^{\prime}}+P_{o}\right) & =\epsilon\left(-i\left(\frac{c_{o}}{T}+1\right)\right)^{-r} F\left(\frac{1}{T}+P_{\theta}\right), \\
F\left(\frac{1}{T+c_{o}}+P_{o}\right) & =\epsilon e^{\pi i r / 2} \frac{\left(i\left(T+c_{\sigma}\right)\right)^{-r}}{(i T)^{-r}} F\left(\frac{1}{T}+P_{o}\right) .
\end{aligned}
$$

Defining $\alpha_{g}$ by $\epsilon e^{\pi i r / 2}=e^{-2 \pi i \alpha_{0}},\left(0 \leqq \alpha_{\theta}<1\right)$, we have

$$
\begin{aligned}
& \exp \left\{\frac{2 \pi i \alpha_{o}}{c_{g}}\left(T+c_{g}\right)\right\}\left(i\left(T+c_{g}\right)\right)^{r} F\left(\frac{1}{T+c_{g}}+P_{o}\right) \\
& =\exp \left\{\frac{2 \pi i \alpha_{o}}{c_{g}} T\right\}(i T)^{r} F\left(\frac{1}{T}+P_{o}\right)
\end{aligned}
$$

and therefore obtain a Fourier expansion

$$
\exp \left\{\frac{2 \pi i \alpha_{o}}{c_{g}} T\right\}(i T)^{r} F\left(\frac{1}{T}+P_{g}\right)=\sum_{m} a_{m}^{(\sigma)} \exp \left\{-\frac{2 \pi i m T}{c_{g}}\right\}
$$

or, going back to the variable $\tau$,

$$
\begin{aligned}
F(\tau) & =\exp \left\{-\frac{2 \pi i \alpha_{g}}{c_{o}} \frac{1}{\tau-P_{o}}\right\}\left(\frac{i}{\tau-P_{o}}\right)^{-r} \sum_{m} a_{m}^{(o)} \exp \left\{-\frac{2 \pi i m}{c_{o}} \frac{1}{\tau-P_{o}}\right\} \\
& =x^{\alpha_{\sigma}}\left(-i\left(\tau-P_{g}\right)\right)^{r} \sum_{m} a_{m}^{(g)} x^{m}
\end{aligned}
$$

with $x=\exp \left\{-2 \pi i /\left(c_{g}\left(\tau-P_{o}\right)\right)\right\}$. We place the restriction on $F(\tau)$ that only a finite number $\mu_{o}$ of terms have negative exponents and write 


$$
\begin{array}{rlrl}
F(\tau) & =\left(-i\left(\tau-P_{\theta}\right)\right)^{r} x^{\alpha_{\theta}} \sum_{m=-\mu_{0}}^{\infty} a_{m}^{(o)} x^{m}=\left(-i\left(\tau-P_{g}\right)\right)^{r} x^{\alpha_{0}} f_{g}(x), \\
x & =\exp \left\{-\frac{2 \pi i}{c_{\theta}\left(\tau-P_{\theta}\right)}\right\}, & g=1,2, \cdots, s .
\end{array}
$$

The functions $f_{\theta}(x)$ are regular inside the unit circle except, when $\mu_{o}>0$, for poles of order $\mu_{g}$ at $x=0$. The functions

$$
P_{g}(x)=a_{-\mu_{\theta}}^{(g)} x^{-\mu_{\theta}}+\cdots+a_{-1}^{(g)} x^{-1}, \quad g=1,2, \cdots, s,
$$

are the principal parts of the $f_{g}(x)$ at $x=0$. We understand $P_{g}(x)$ to be zero if $\mu_{0}=\mathbf{0}$.

In the following sections we shall determine the $a_{m}^{(0)}$ in terms of the constants of (1.11) and (3.13). The functions $F^{*}(\tau)$ of (1.32) are then found as follows. We consider the point $A / C$ where $A$ and $C$ are the coefficients of $\tau$ in (1.31). If $C \neq 0$ we take $C>0$ and the point is a real rational point. If $C=0$ the point is the point at infinity. In either case $A / C$ is congruent to some parabolic vertex $P_{l}$ of $R$, where $l$ is determined by $A / C$ and hence by the transformation. Therefore we can find a transformation

$$
\tau^{\prime}=\frac{a_{1} \tau+b_{1}}{c_{1} \tau+d_{1}}, \quad c_{1} \geqq 0,
$$

of $\gamma$ which takes $P_{0}$ into the point $A / C$. That is

hence

$$
\frac{a_{1} p_{0}+b_{1} q_{0}}{c_{1} p_{0}+d_{1} q_{0}}=\frac{A}{C}
$$

$$
a_{1} p_{0}+b_{1} q_{0}={ }_{\kappa} A, \quad c_{1} p_{0}+d_{1} q_{0}={ }_{\kappa} C .
$$

Solving these equations for $p_{o}$ and $q_{\theta}$, we have

$$
p_{0}=\kappa\left(A d_{1}-C b_{1}\right), \quad q_{0}=\kappa\left(C a_{1}-A c_{1}\right) ;
$$

then, since $\left(p_{0}, q_{\theta}\right)=1$, we have $\kappa= \pm 1$. Using these equations and the fact that the determinant of (3.21) is unity, we find

$$
\frac{A \tau+B}{C \tau+D}=\frac{a_{1}\left(\frac{\dot{\kappa} p_{0} \tau+d_{1} B-b_{1} D}{\kappa q_{0} \tau+a_{1} D-c_{1} B}\right)+b_{1}}{c_{1}\left(\frac{\kappa p_{0} \tau+d_{1} B-b_{1} D}{\kappa q_{0} \tau+a_{1} D-c_{1} B}\right)+d_{1}}
$$

then, by (1.11), we obtain the relation 


$$
\begin{aligned}
F\left(\frac{A \tau+B}{C \tau+D}\right)= & \epsilon\left(a_{1}, b_{1}, c_{1}, d_{1}\right)\left(-i\left(c_{1}\left(\frac{\kappa p_{0} \tau+d_{1} B-b_{1} D}{\kappa q_{\rho} \tau+a_{1} D-c_{1} B}\right)+d_{1}\right)\right)^{-r} \\
& \cdot F\left(\frac{\kappa p_{0} \tau+d_{1} B-b_{1} D}{\kappa q_{\rho} \tau+a_{1} D-c_{1} B}\right) .
\end{aligned}
$$

Now applying (3.12) to the function on the right and simplifying, we obtain

$$
\begin{aligned}
& F\left(\frac{A \tau+B}{C \tau+D}\right)=\epsilon\left(a_{1}, b_{1}, c_{1}, d_{1}\right) \exp \left\{\kappa \frac{\pi i r}{2}\right\} \exp \left\{\kappa \frac{2 \pi i q_{0}}{c_{0}} \alpha_{0}\left(a_{1} D-c_{1} B\right)\right\} \\
& \cdot(-i(C \tau+D))^{-r} q_{\sigma}^{-r} \exp \left\{\frac{2 \pi i q_{\theta}^{2}}{c_{g}} \alpha_{\theta} \tau\right\} \\
& \cdot \sum_{m=-\mu_{\theta}}^{\infty} a_{m}^{(\sigma)} \exp \left\{\kappa \frac{2 \pi i q_{\sigma}}{c_{\sigma}} m\left(a_{1} D-c_{1} B\right)\right\} \exp \left\{\frac{2 \pi i q_{\theta}^{2}}{c_{\theta}} m \tau\right\} \\
& =\epsilon\left(a_{1}, b_{1}, c_{1}, d_{1}\right) \exp \left\{\kappa \frac{\pi i r}{2}\right\} \exp \left\{\kappa \frac{2 \pi i q_{0}}{c_{0}} \alpha_{0}\left(a_{1} D-c_{1} B\right)\right\} \\
& \cdot(-i(C \tau+D))^{-r} q_{\sigma}^{-r} \exp \left\{\frac{2 \pi i q_{\theta}^{2}}{c_{g}} \alpha_{\theta} \tau\right\} \\
& \cdot f_{0}\left(\exp \left\{\kappa \frac{2 \pi i q_{0}}{c_{0}}\left(a_{1} D-c_{1} B\right)\right\}^{\cdot} \exp \left\{\frac{2 \pi i q_{0}^{2}}{c_{0}} \tau\right\}\right)
\end{aligned}
$$

therefore we have

$$
\begin{aligned}
F^{*}(\tau)= & q_{0}^{-r} \exp \left\{\frac{2 \pi i q_{\theta}^{2}}{c_{\theta}} \alpha_{\theta} \tau\right\} \\
& \cdot f_{0}\left(\exp \left\{\kappa \frac{2 \pi i q_{\theta}}{c_{\theta}}\left(a_{1} D-c_{1} B\right)\right\} \exp \left\{\frac{2 \pi i q_{\theta}^{2}}{c_{\theta}} \tau\right\}\right), \\
\epsilon^{*}= & \epsilon\left(a_{1}, b_{1}, c_{1}, d_{1}\right) \exp \left\{\kappa \frac{\pi i r}{2}\right\} \exp \left\{\kappa \frac{2 \pi i q_{\theta}}{c_{\theta}} \alpha_{\theta}\left(a_{1} D-c_{1} B\right)\right\} .
\end{aligned}
$$

4. The transformation equation. In order to make use of the transformation equation (1.11) in the next section we shall need it in a special form. We consider all rational real nonnegative numbers $h / k,(h, k)=1, k>0$, $h \geqq 0$. Then by (3.12) we have, with $\tau=P_{o}-k /\left(c_{g}(i z+h)\right)$,

$$
\begin{aligned}
f_{0}\left(\exp \left\{2 \pi i\left(\frac{h}{k}+\frac{i z}{k}\right)\right\}\right)= & \exp \left\{-2 \pi i \alpha_{0}\left(\frac{h}{k}+\frac{i z}{k}\right)\right\} \\
& \cdot\left(\frac{k}{c_{0}(z-i h)}\right)^{-r} F\left(P_{0}-\frac{k}{c_{0}(i z+h)}\right) .
\end{aligned}
$$


Now $P_{g}-k / c_{g} h$ is a real rational point and hence is congruent under $\gamma$ to some parabolic vertex $P_{\beta}$ of $R$ where $\beta=\beta(h, k, g)$. Then there is a transformation of $\gamma$,

$$
\tau^{\prime}=\frac{a \tau+b}{c \tau+d}
$$

where $a, b, c$, and $d$ are determined, although not uniquely, by $h, k$, and $g$, which takes $P_{o}-k / c_{g} h$ into $P_{\beta}$, that is,

$$
\frac{p_{\beta}}{q_{\beta}}=\left\{a\left(\frac{p_{0}}{q_{0}}-\frac{k}{c_{0} h}\right)+b\right\}\left\{c\left(\frac{p_{0}}{q_{0}}-\frac{k}{c_{0} h}\right)+d\right\}^{-1} .
$$

We have seen that $q_{\theta}^{2}$ divides $c_{0}$, so we have

$$
\begin{aligned}
& a\left(\frac{c_{0}}{q_{0}} p_{\theta} h-k\right)+b c_{0} h=\sigma_{h, k}^{(0)} p_{\beta}, \\
& c\left(\frac{c_{0}}{q_{0}} p_{0} h-k\right)+d c_{0} h=\sigma_{h, k}^{(0)} q_{\beta} .
\end{aligned}
$$

From these equations and the fact that $h$ and $k$ are relatively prime, it follows that $\sigma_{h, t}^{(o)}$ divides $c_{\theta}$, which implies

$$
-c_{o} \leqq \sigma_{h, k}^{(o)} \leqq c_{o}, \quad \sigma_{h, k}^{(o)} \neq 0 .
$$

Using this transformation we have by (1.11)

$$
\begin{aligned}
F\left(P_{0}-\frac{k}{c_{o}(i z+h)}\right)= & \left(\epsilon_{h, k}^{(o)}\right)^{-1}\left(-i\left(c P_{0}-\frac{c k}{c_{0}(i z+h)}+d\right)\right)^{r} \\
& \cdot F\left(\frac{a\left(P_{o}-\frac{k}{c_{o}(i z+h)}\right)+b}{c\left(P_{o}-\frac{k}{c_{o}(i z+h)}\right)+d}\right) \\
= & \left(\epsilon_{h, k}^{(o)}\right)^{-1}\left(-i \frac{c_{o}\left(c P_{o}+d\right) i z+\sigma_{h, k}^{(o)} q_{\beta}}{c_{o}(i z+h)}\right)^{r} \\
& \cdot F\left(P_{\beta}+\frac{k c_{o} i z}{\sigma_{h, k}^{(o)} q_{\beta}\left(c_{o}\left(c P_{o}+d\right) i z+\sigma_{h, k}^{(o)} q_{\beta}\right)}\right),
\end{aligned}
$$

where $\epsilon_{h, \mathbf{k}}^{(0)}=\epsilon(a, b, c, d)$, the $\epsilon$ of (1.11) associated with the transformation determined by (4.12). We now apply the Fourier expansion (3.12) to the function on the right in (4.15), combine it with (4.11), and obtain after some simplification 


$$
\begin{aligned}
& f_{0}\left(\exp \left\{2 \pi i\left(\frac{h}{k}+i z\right)\right\}\right) \\
&=\left(\epsilon_{h, k}^{(o)}\right)^{-1} \exp \left\{\left(1-\delta_{h, k}^{(o)}\right) \frac{\pi i r}{2}\right\} \\
& \cdot \exp \left\{-\frac{2 \pi i}{k}\left(\alpha_{\theta} h+\frac{\sigma_{h, k}^{(o)} q_{\beta}\left(c P_{o}+d\right) \alpha_{\beta}}{c_{\beta}}\right)\right\} \\
& \cdot\left(\frac{c_{\sigma} z}{\left|\sigma_{h, k}^{(o)}\right| q_{\beta}}\right)^{r} \exp \left\{\frac{2 \pi}{k}\left(\alpha_{\sigma} z-\frac{\alpha_{\beta}\left(\sigma_{h, k}^{(o)}\right)^{2} q_{\beta}^{2}}{c_{\beta} c_{o}} \frac{1}{z}\right)\right\} \\
& \cdot f_{\beta}\left(\exp \left\{-\frac{2 \pi}{k c_{\beta}}\left(\sigma_{h, k}^{(o)} q_{\beta}\left(c P_{o}+d\right) i+\frac{\left(\sigma_{h, k}^{(o)}\right)^{2} q_{\beta}{ }^{2}}{c_{o}} \frac{1}{z}\right)\right\}\right)
\end{aligned}
$$

where $\delta_{h, \mathrm{k}}^{(\rho)}=-1$ if $\sigma_{h, \mathrm{k}}^{(\rho)}>0$ and $\delta_{h, \mathrm{k}}^{(\rho)}=1$ if $\sigma_{h, \mathrm{k}}^{(\rho)}<0$. The factor involving $\delta_{h, \mathrm{k}}^{(\rho)}$ arises because we have combined three factors into the single factor $\left(c_{g} z /\left(\left|\sigma_{h, \mathbf{k}}^{(o)}\right| q_{\beta}\right)\right)$.

In order to simplify the notation we write (4.2) as

$$
\begin{aligned}
& f_{o}\left(\exp \left\{2 \pi i\left(\frac{h}{k}+i z\right)\right\}\right) \\
& \quad=\Omega_{h, k}^{(o)} \Psi_{h, k}^{(o)}(z) f_{\beta}\left(\exp \left\{G_{h, k}^{(o)} i-\frac{2 \pi\left(\dot{\sigma}_{h, k}^{(o)}\right)^{2} q_{\beta}{ }^{2}}{k c_{\beta} c_{o}} \frac{1}{z}\right\}\right), \beta=\beta(h, k, g),
\end{aligned}
$$

where

$$
\begin{aligned}
\Omega_{h, k}^{(o)}= & \left(\epsilon_{h, k}^{(o)}\right)^{-1} \exp \left\{\left(1-\delta_{h, k}^{(b)}\right) \frac{\pi i r}{2}\right\} \\
& \cdot \exp \left\{-\frac{2 \pi i}{k}\left(\alpha_{o} h+\frac{\sigma_{h, k} q_{\beta}\left(c P_{o}+d\right) \alpha_{\beta}}{c_{\beta}}\right)\right\}, \\
\Psi_{h, k}^{(o)}(z)= & \left(\frac{c_{o} z}{\left|\sigma_{h, k}^{(o)}\right| q_{\beta}}\right)^{r} \exp \left\{\frac{2 \pi}{k}\left(\alpha_{o} z-\frac{\alpha_{\beta}\left(\sigma_{h, k}^{(o)}\right)^{2} q_{\beta}{ }^{2}}{c_{\beta} c_{o}} \frac{1}{z}\right)\right\}, \\
G_{h, k}^{(o)}= & -\frac{2 \pi}{k c_{\beta}} \sigma_{h, k}^{(o)} q_{\beta}\left(c P_{o}+d\right) .
\end{aligned}
$$

We note that $\left|\Omega_{h, k}^{(o)}\right|=1$ and that $G_{h, k}^{(o)}$ is real.

5. A convergent series for $a_{n}{ }^{\left({ }^{\circ}\right)}$. In the following we shall let $\sum_{h, \mathrm{k}}^{N}$ designate a sum over all $h$ and $k$ such that $0 \leqq h<k \leqq N$ and $(h, k)=1$.

We let $N$ be a positive integer and have by Cauchy's theorem

$$
a_{n}^{(g)}=\frac{1}{2 \pi i} \int \frac{f_{o}(x)}{x^{n+1}} d x
$$


where we take the integral over the circle $|x|=e^{-2 \pi N^{-2}}$ in the positive direction. We make the usual Farey dissection, of order $N$, of the circle and set

$$
x=\exp \left\{-2 \pi N^{-2}+2 \pi i(h / k)+2 \pi i \phi\right\}
$$

on the arc corresponding to $h / k$. We then have

$$
\begin{aligned}
a_{n}^{(\theta)}= & \sum_{h, k}^{N} \int_{-\theta^{\prime}}^{\theta^{\prime \prime}} \frac{f_{\theta}\left(\exp \left\{-2 \pi N^{-2}+2 \pi i(h / k)+2 \pi i \phi\right\}\right)}{\exp \left\{-2 \pi N^{-2} n+2 \pi i(n h / k)+2 \pi i n \phi\right\}} d \phi \\
= & \exp \left\{2 \pi N^{-2} n\right\} \sum_{h, k}^{N} \exp \left\{-2 \pi i n \frac{h}{k}\right\} \\
& \cdot \int_{-\theta^{\prime}}^{\theta^{\prime \prime}} f_{0}\left(\exp \left\{2 \pi i\left(\frac{h}{k}+\frac{i k\left(N^{-2}-i \phi\right)}{k}\right)\right\}\right) \exp \{-2 \pi i n \phi\} d \phi
\end{aligned}
$$

where $\theta^{\prime}$ and $\theta^{\prime \prime}$, which depend on $h$ and $k$, determine the end points of the Farey arcs. Equation (4.31) now yields the result

$$
\begin{aligned}
a_{n}^{(\theta)}= & \exp \left\{2 \pi N^{-2} n\right\} \sum_{h, k}^{N} \Omega_{h, k}^{(\theta)} \exp \left\{-2 \pi i n \frac{h}{k}\right\} \int_{-\theta^{\prime}}^{\theta^{\prime \prime}} \Psi_{h, k}^{(\theta)}\left(k\left(N^{-2}-i \phi\right)\right) \\
& \cdot f_{\beta}\left(\exp \left\{G_{h, k}^{(\theta)} i-\frac{2 \pi\left(\sigma_{h, k}^{(\theta)}\right)^{2} q_{\beta^{2}}}{k c_{\beta} c_{\sigma}} \frac{1}{k\left(N^{-2}-i \phi\right)}\right\}\right) \exp \{-2 \pi i n \phi\} d \phi
\end{aligned}
$$

with $\beta=\beta(h, k, g)$.

Now $f_{\beta}(x)$ is dominated by its principal part $P_{\beta}(x)$ near $x=0$; so we put

$$
D_{\beta}(x)=f_{\beta}(x)-P_{\beta}(x)=\sum_{m=0}^{\infty} a_{m}^{(\beta)} x^{m}
$$

and split (5.1) into two parts

$$
a_{n}^{(0)}=Q^{(0)}(n)+R^{(0)}(n),
$$

where

$$
\begin{aligned}
Q^{(o)}(n)= & \exp \left\{2 \dot{\pi} N^{-2} n\right\} \sum_{h, k}^{N} \Omega_{h, k}^{(o)} \exp \left\{-2 \pi i n \frac{h}{k}\right\} \\
& \int_{-\theta^{\prime}}^{\theta^{\prime \prime}} \Psi_{h, k}^{(o)}\left(k\left(N^{-2}-i \phi\right)\right) \\
\cdot & P_{\beta}\left(\exp \left\{G_{h, k}^{(o)} i-\frac{2 \pi\left(\sigma_{h, k}^{(o)}\right)^{2} q_{\beta}{ }^{2}}{k c_{\beta} c_{o}} \frac{1}{k\left(N^{-2}-i \phi\right)}\right\}\right) \exp \{-2 \pi i n \phi\} d \phi
\end{aligned}
$$

and where $R^{(0)}(n)$ is given by 


$$
\begin{aligned}
R^{(\theta)}(n)=\exp \left\{2 \pi N^{-2} n\right\} \sum_{h, k}^{N} \Omega_{h, k}^{(\theta)} \exp \left\{-2 \pi i n \frac{h}{k}\right\} \int_{-\theta^{\prime}}^{\theta^{\prime \prime}} \Psi_{h, k}^{(o)}\left(k\left(N^{-2}-i \phi\right)\right) \\
\cdot D_{\beta}\left(\exp \left\{G_{h, k}^{(\theta)} i-\frac{2 \pi\left(\sigma_{h, k}^{(\theta)}\right)^{2} q_{\beta}^{2}}{k c_{\beta} c_{\theta}} \frac{1}{k\left(N^{-2}-i \phi\right)}\right\}\right) \\
\cdot \exp \{-2 \pi i n \phi\} d \phi .
\end{aligned}
$$

We can now make an estimate of $R^{(\theta)}(n)$. From the theory of Farey fractions we have

$$
\frac{1}{2 k N}<\theta^{\prime}<\frac{1}{k N}, \quad \frac{1}{2 k N}<\theta^{\prime \prime}<\frac{1}{k N}
$$

hence for $-\theta^{\prime} \leqq \phi \leqq \theta^{\prime \prime}$ we have

$$
\begin{gathered}
R\left(k\left(N^{-2}-i \phi\right)\right)=k N^{-2}, \quad R\left(\frac{1}{k\left(N^{-2}-i \phi\right)}\right) \geqq \frac{k}{2}, \\
\left|k\left(N^{-2}-i \phi\right)\right| \leqq 2^{1 / 2} N^{-1} .
\end{gathered}
$$

Using these results we find, by (4.33),

$$
\begin{aligned}
& \left|\Psi_{h, k}^{(o)}\left(k\left(N^{-2}-i \phi\right)\right) D_{\beta}\left(\exp \left\{G_{h, k}^{(g)} i-\frac{2 \pi\left(\sigma_{h, k}^{(o)}\right)^{2} q_{\beta}^{2}}{k c_{\beta} c_{o}} \frac{1}{k\left(N^{-2}-i \phi\right)}\right\}\right)\right| \\
& \leqq \frac{c_{\sigma}^{r}}{\left|\sigma_{h, k}^{(0)}\right|^{r} q_{\beta}^{r}} 2^{r / 2} N^{-r} \exp \left\{2 \pi \alpha_{g} N^{-2}-\frac{\pi \alpha_{\beta}\left(\begin{array}{c}
(\sigma) \\
\left.\sigma_{h, k}\right)^{2} q_{\beta}^{2}
\end{array}\right.}{c_{\beta} c_{g}}\right\} \\
& \cdot \sum_{m=0}^{\infty}\left|a_{m}^{(\beta)}\right| \exp \left\{-\frac{\pi\left(\sigma_{h, k}^{(0)}\right)^{2} q_{\beta}^{2}}{c_{\beta} c_{o}} m\right\} \\
& \leqq \frac{c_{g}^{r}}{q_{\beta}^{r}} 2^{r / 2} N^{-r} \exp \left\{2 \pi \alpha_{0} N^{-2}\right\} \sum_{m=0}^{\infty}\left|a_{m}^{(\beta)}\right| \exp \left\{-\frac{\pi q_{\beta}^{2}}{c_{\beta} c_{g}} m\right\} \\
& \leqq K N^{-r} \exp \left\{2 \pi \alpha_{0} N^{-2}\right\}
\end{aligned}
$$

where we have used (4.14) and the fact that the series for $D_{\beta}(x)$ converges for $|x|<1$. Combining this result with (5.23), we have

$$
\begin{aligned}
\left|R^{(\theta)}(n)\right| \leqq & \exp \left\{2 \pi N^{-2} n\right\} K N^{-r} \exp \left\{2 \pi \alpha_{0} N^{-r}\right\} \\
& \cdot \sum_{h, k}^{N} \int_{-\theta^{\prime}}^{\theta^{\prime \prime}} d \phi=K N^{-r} \exp \left\{2 \pi\left(n+\alpha_{0}\right) N^{-2}\right\} .
\end{aligned}
$$

The determination of $a_{n}^{(0)}$ now rests entirely on the evaluation of $Q^{(\theta)}(n)$. To accomplish this we set $w=N^{-2}-i \phi$ in (5.22) and have, by (3.13), the relation 


$$
\begin{aligned}
Q^{(\theta)}(n)= & \exp \left\{2 \pi N^{-2} n\right\} \sum_{h, k}^{N} \Omega_{h, k}^{(o)} \exp \left\{-2 \pi i n \frac{h}{k}\right\} \\
& \cdot \int_{N^{-2}+i \theta^{\prime}}^{N^{-2}-i \theta^{\prime \prime}} i \Psi_{h, k}^{(o)}(k w) \sum_{\nu=1}^{\mu_{\beta}} a_{-\nu}^{(\beta)} \\
& \cdot \exp \left\{-\nu G_{h, k}^{(o)} i+\frac{2 \pi\left(\sigma_{h, k}^{(o)}\right)^{2} q_{\beta}^{2}}{k c_{\beta} c_{o}} \frac{\nu}{k w}\right\} \exp \left\{2 \pi n w-2 \pi n N^{-2}\right\} d w \\
= & \sum_{h, k}^{N} \Omega_{h, k}^{(o)} \exp \left\{-2 \pi i n \frac{h}{k}\right\} \sum_{\nu=1}^{\mu_{\beta}} a_{->}^{(\beta)} \exp \left\{-\nu G_{h, k}^{(o)}\right\} I_{h, k, \nu}^{(o)}(n),
\end{aligned}
$$

where

$$
I_{h, k, \nu}^{(o)}(n)=\frac{1}{i} \int_{N^{-2}-i \theta^{\prime \prime}}^{N^{-2}+i \theta \prime} \Psi_{h, k}^{(o)}(k w) \exp \left\{\frac{2 \pi \nu\left(\sigma_{h, k}^{(o)}\right)^{2} q_{\beta}{ }^{2}}{k^{2} c_{\beta} c_{0} w}+2 \pi n w\right\} d w
$$

and where the inner sum is to be taken as zero if $\mu_{\beta}=0$. By (4.33) we may write (5.52) as

$$
\begin{aligned}
I_{h, k, \nu}^{(o)}(n)= & k^{r}\left(\frac{c_{\sigma}}{\left|\sigma_{h, k}^{(o)}\right| q_{\beta}}\right)^{r} \frac{1}{i} \\
& \cdot \int_{N^{-2}-i \theta^{\prime \prime}}^{N^{-2}+i \theta^{\prime}} w^{r} \exp \left\{2 \pi\left(\alpha_{\theta}+n\right) w+\frac{2 \pi\left(\sigma_{h, k}^{(o)}\right)^{2} q_{\beta}^{2}}{k^{2} c_{\beta} c_{o} w}\left(\nu-\alpha_{\beta}\right)\right\} .
\end{aligned}
$$

We restrict our considerations to those $a_{n}^{(\theta)}$ for which $\alpha_{0}+n>0$. That is, we leave undetermined all $a_{0}^{(0)}$ for which $\alpha_{0}=0$ but determine all other $a_{n}^{(o)}$. This restriction is necessary in order that a certain integral shall converge. We cut the $w$-plane from 0 to $-\infty$ along the negative real axis and consider a path of integration encircling the cut in the positive sense and connecting the points

$$
-\infty,-\epsilon,-\epsilon-i \theta^{\prime \prime}, N^{-2}-i \theta^{\prime \prime}, N^{-2}+i \theta^{\prime},-\epsilon+i \theta^{\prime},-\epsilon,-\infty
$$

by straight lines where we take $0<\epsilon<N^{-2}$. Then we can write

$$
\begin{aligned}
& \vec{k}\left(\frac{c_{\theta}}{\left|\sigma_{h, k}^{(0)}\right| q_{\beta}}\right)^{-r} I_{h, k, \nu}^{(o)}(n)=\frac{1}{i} \int_{-\infty}^{\left(0^{+}\right)}-\frac{1}{i} \int_{-\infty}^{-\epsilon}-\frac{1}{i} \int_{-\epsilon}^{-\epsilon-i \theta^{\prime \prime}} \\
&-\frac{1}{i} \int_{-\epsilon-i \theta^{\prime \prime}}^{N^{-2}-i \theta^{\prime \prime}}-\frac{1}{i} \int_{N^{-2}+i \theta^{\prime}}^{-\epsilon+i \theta^{\prime}} \\
&-\frac{1}{i} \int_{-\epsilon+i \theta^{\prime}}^{-\epsilon}-\frac{1}{i} \int_{-\epsilon}^{-\infty} \\
&= L_{h, k, \nu}^{(o)}(n)-J_{1}-J_{2}-J_{3}-J_{4}-J_{5}-J_{6},
\end{aligned}
$$


say. All these integrals have the same integrand

$$
w^{r} \exp \left\{2 \pi\left(\alpha_{\theta}+n\right) w+\frac{2 \pi\left(\sigma_{h, k}^{(o)}\right)^{2} q_{\beta}^{2}}{k^{2} c_{\beta} c_{0} w}\left(\nu-\alpha_{\beta}\right)\right\} .
$$

The integral $J_{1}$ is to be taken on the border below the cut, $J_{6}$ above the cut. In the integral $J_{2}$ we have $w=-\epsilon+i v, 0 \geqq v \geqq-\theta^{\prime \prime}$, and

$$
R(w)=-\epsilon, \quad R(1 / w)=-\epsilon /\left(\epsilon^{2}+v^{2}\right)<0, \quad|w| \leqq 2^{1 / 2} k^{-1} N^{-1},
$$

and therefore

$$
\left|J_{2}\right| \leqq \theta^{\prime \prime} 2^{r / 2} k^{-r} N^{-r}<2^{r / 2} k^{-r-1} N^{-r-1} .
$$

Similarly we have

$$
\left|J_{5}\right| \leqq 2^{r / 2} k^{-r-1} N^{-r-1} \text {. }
$$

In the integral $J_{8}$ we have $w=u-i \theta^{\prime \prime},-N^{-2}<-\epsilon \leqq u \leqq N^{-2}$, and

$$
R(w)=u \leqq N^{-2}, \quad R(1 / w) \leqq 4 k^{2}, \quad|w| \leqq 2^{1 / 2} k^{-1} N^{-1}
$$

and therefore, using (4.14),

$$
\left|J_{3}\right| \leqq\left(N^{-2}+\epsilon\right) 2^{r / 2} k^{-r} N^{-r} \exp \left\{2 \pi\left(\alpha_{o}+n\right) N^{-2}+\frac{8 \pi\left(\sigma_{h, k}^{(o)}\right)^{2} q_{\beta}^{2}}{c_{\theta} c_{\beta}}\left(\nu-\alpha_{\beta}\right)\right\}
$$

$$
\begin{aligned}
& \leqq 2 N^{-2} 2^{r / 2} k^{-r} N^{-r} \exp \left\{2 \pi\left(\alpha_{\theta}+n\right) N^{-2}+\frac{8 \pi c_{o} q q^{2}\left(\mu_{\theta}-\alpha_{\beta}\right)}{c_{\beta}}\right\} \\
& \leqq K k^{-r-1} N^{-r-1} \exp \left\{2 \pi\left(\alpha_{\theta}+n\right) N^{-2}\right\}
\end{aligned}
$$

and similarly

$$
\left|J_{4}\right| \leqq K k^{-r-1} N^{-r-1} \exp \left\{2 \pi\left(\alpha_{0}+n\right) N^{-2}\right\} .
$$

Finally we have

$$
\begin{aligned}
& J_{1}+J_{6}=\frac{1}{i} \int_{-\infty}^{-\epsilon}|w|^{r} \exp \{-\pi i r\} \\
& \exp \left\{2 \pi\left(\alpha_{o}+n\right) w+\frac{2 \pi\left(\sigma_{h, k}^{(o)}\right)^{2} q_{\beta}{ }^{2}\left(\nu-\alpha_{\beta}\right)}{k^{2} c_{\beta} c_{o} w}\right\} d w \\
& +\frac{1}{i} \int_{-\epsilon}^{-\infty}|w|^{r} \exp \{\pi i r\} \\
& \cdot \exp \left\{2 \pi\left(\alpha_{o}+n\right) w+\frac{2 \pi\left(\sigma_{h, k}^{(o)}\right)^{2} q_{\beta}^{2}\left(\nu-\alpha_{\beta}\right)}{k^{2} c_{\beta} c_{\sigma} w}\right\} d w . \\
& =-2 \sin \pi r \int_{\epsilon^{2}}^{\infty} \exp \left\{-2 \pi\left(\alpha_{0}+n\right) t-\frac{2 \pi\left(\sigma_{h, k}^{(\sigma)}\right)^{2} q_{\beta}{ }^{2}\left(\nu-\alpha_{\beta}\right)}{k^{2} c_{\beta} c_{0} t}\right\} d t
\end{aligned}
$$


where our restriction $\alpha_{0}+n>0$ ensures the convergence of the integrals. Combining (5.61), (5.62), (5.63), (5.64), (5.65), (5.66), and letting $\epsilon \rightarrow 0$, we have

$$
\begin{aligned}
I_{h, k, \nu}^{(o)}(n)= & k^{r}\left(\frac{c_{\sigma}}{\left|\sigma_{h, k}^{(0)}\right| q_{\beta}}\right)^{r} L_{h, k, \nu}^{(\theta)}(n)+2 k^{r}\left(\frac{c_{\theta}}{\left|\sigma_{h, k}^{(o)}\right| q_{\beta}}\right)^{r} \\
& \cdot \sin \pi r \int_{0}^{\infty} t^{r} \exp \left\{-2 \pi\left(\alpha_{\theta}+n\right) t-\frac{2 \pi\left(\sigma_{h, k}^{(o)}\right)^{2} q_{\beta}{ }^{2}\left(\nu-\alpha_{\beta}\right)}{k^{2} c_{\beta} c_{\theta} t}\right\} d t \\
& +O\left(k^{r}\left(\frac{c_{\sigma}}{\left|\sigma_{h, k}^{(o)}\right| q_{\beta}}\right)^{r} k^{-r-1} N^{-r-1} \exp \left\{2 \pi\left(\alpha_{\theta}+n\right) N^{-2}\right\}\right) .
\end{aligned}
$$

Introducing this into (5.51) and using (4.14), we obtain

$$
\begin{aligned}
Q^{(\theta)}(n)= & \sum_{h, k}^{N} \Omega_{h, k}^{(\theta)} \exp \left\{-2 \pi i n \frac{h}{k}\right\} \sum_{\nu=1}^{\mu_{\beta}} a_{\rightarrow,}^{(\beta)} \exp \left\{-\nu G_{h, k}^{(o)} i\right\} \\
& \cdot k^{r}\left(\frac{c_{\sigma}}{\left|\sigma_{h, k}^{(o)}\right| q_{\beta}}\right)^{r}\left\{L_{h, k, \nu}^{(o)}(n)+M_{h, k, \nu}^{(o)}(n)\right\} \\
& +O\left(N^{-r-1} \exp \left\{2 \pi\left(\alpha_{\theta}+n\right) N^{-2}\right\} \sum_{\nu=1}^{\mu_{\beta}}\left|\alpha_{-, \nu}^{(\beta)}\right| \sum_{h, k}^{N} k^{-1}\right)
\end{aligned}
$$

with

$$
L_{h, k, \nu}^{(o)}(n)=\frac{1}{i} \int_{-\infty}^{\left(0^{+}\right)} w^{r} \exp \left\{2 \pi\left(\alpha_{0}+n\right) w+\frac{2 \pi\left(\sigma_{h, k}^{(o)}\right)^{2} q_{\beta}^{2}\left(\nu-\alpha_{\beta}\right)}{k^{2} c_{\beta} c_{0} w}\right\} d w
$$

and

(5.73) $M_{h, k, \nu}^{(0)}(n)=2 \sin \pi r \int_{0}^{\infty} t^{r} \exp \left\{-2 \pi\left(\alpha_{0}+n\right) t-\frac{2 \pi\left(\sigma_{h, k}^{(o)}\right)^{2} q_{\beta}{ }^{2}\left(\nu-\alpha_{\beta}\right)}{k^{2} c_{\beta} c_{0} t}\right\} d t$

Now since $\sum_{h, k}^{N} k^{-1} \leqq N$, we have, by (5.71), (5.21), and (5.4),

$$
\begin{aligned}
& a_{n}^{(o)}=\sum_{h, k}^{N} \Omega_{h, k}^{(o)} \exp \left\{-2 \pi i n \frac{h}{k}\right\} k^{r}\left(\frac{c_{\sigma}}{\left|\sigma_{h, k}^{(o)}\right| q_{\beta}}\right)^{r} \sum_{\nu=1}^{\mu_{\beta}} a_{-\nu}^{(\beta)} \\
& \cdot \exp \left\{-\nu G_{h, k}^{(o)} i\right\}\left\{L_{h, k, \nu}^{(o)}(n)+M_{h, k, \nu}^{(o)}(n)\right\} \\
&+O\left(N^{-r} \exp \left\{2 \pi\left(\alpha_{o}+n\right) N^{-2}\right\}\right) .
\end{aligned}
$$

If we keep $n$ fixed and let $N$ tend to infinity, the error term tends to zero, since $r>0$. Hence the series thus obtained converges and we have

$$
\begin{aligned}
a_{n}^{(o)}= & \sum_{h, k}^{\infty} \Omega_{h, k}^{(o)} \exp \left\{-2 \pi i n \frac{h}{k}\right\} k^{r}\left(\frac{c_{o}}{\left|\sigma_{h, k}^{(o)}\right| q_{\beta}}\right)^{r} \\
& \cdot \sum_{\nu=1}^{\mu_{\beta}} a_{-,}^{(\beta)} \exp \left\{-\nu G_{h, k}^{(o)} i\right\}\left\{L_{h, k, \nu}^{(o)}(n)+M_{h, k, \nu}^{(o)}(n)\right\} .
\end{aligned}
$$


6. Evaluation of the integrals. We now express $L_{k, n, v}^{(0)}(n)$ and $M_{n, k, \nu}^{(0)}(n)$ in terms of Bessel functions. In (5.72) the change of variable $u=2 \pi\left(\alpha_{0}+n\right) w$ yields

$$
\begin{aligned}
L_{h, k, \eta}^{(0)}(n)= & \frac{1}{i\left(2 \pi\left(\alpha_{0}+n\right)\right)^{r+1}} \\
& \cdot \int_{-\infty}^{\left(0^{+}\right)} u^{r} \exp \left\{u+\frac{4 \pi^{2}\left(\sigma_{h, k}^{(0)}\right)^{2} q_{\beta}{ }^{2}\left(\nu-\alpha_{\beta}\right)\left(\alpha_{0}+n\right)}{k^{2} c_{\beta} c_{\theta} u}\right\} d u ;
\end{aligned}
$$

hence we have, by a well known formula,*

$$
\begin{aligned}
L_{h, k, 0}^{(o)}(n)= & \frac{2 \pi\left|\sigma_{h, k}^{(o)}\right|^{r+1} q_{\beta}^{r+1}\left(\nu-\alpha_{\beta}\right)^{(r+1) / 2}}{k^{r+1} c_{\beta}^{(r+1) / 2} c_{o}^{(r+1) / 2}\left(\alpha_{o}+n\right)^{(r+1) / 2}} \\
& \cdot I_{-r-1}\left(\frac{4 \pi\left|\sigma_{h, k}^{(o)}\right| q_{\beta}\left(\left(\nu-\alpha_{\beta}\right)\left(\alpha_{o}+n\right)\right)^{1 / 2}}{k\left(c_{\beta} c_{\theta}\right)^{1 / 2}}\right),
\end{aligned}
$$

where $I_{m}(z)$ is the Bessel function of the first kind with purely imaginary argument.

In (5.73) we set $v=2 \pi\left(\alpha_{v}+n\right) t$ and have

$$
\begin{aligned}
M_{h, k, \nu}^{(o)}(n)= & \frac{2 \sin \pi r}{\left(2 \pi\left(\alpha_{\theta}+n\right)\right)^{r+1}} \\
& \cdot \int_{0}^{\infty} v^{r} \exp \left\{-v-\frac{4 \pi^{2}\left(\sigma_{h, k}^{(o)}\right)^{2} q_{\beta}{ }^{2}\left(\nu-\alpha_{\beta}\right)\left(\alpha_{o}+n\right)}{k^{2} c_{\beta} c_{\theta} v}\right\} d v \\
= & \frac{4\left|\sigma_{h, k}^{(o)}\right|^{r+1} q_{\beta}^{r+1}\left(\nu-\alpha_{\beta}\right)^{(r+1) / 2} \sin \pi r}{k^{r+1} c_{\beta}^{(r+1) / 2} c_{o}^{(r+1) / 2}\left(\alpha_{\theta}+n\right)^{(r+1) / 2}} \\
& \cdot K_{-r-1}\left(\frac{4 \pi\left|\sigma_{h, k}^{(o)}\right| q_{\beta}\left(\left(\nu-\alpha_{\beta}\right)\left(\alpha_{\theta}+n\right)\right)^{1 / 2}}{k\left(c_{\beta} c_{\theta}\right)^{1 / 2}}\right),
\end{aligned}
$$

where $K_{m}(z)$ is the Bessel function of the third kind with purely imaginary argument. $\dagger$ Now adding (6.1) and (6.2) and simplifying by means of the formula

$$
\sin \pi \nu K_{\nu}(z)+\frac{\pi}{2} I_{\nu}(z)=\frac{\pi}{2} I_{-\nu}(z),
$$

we find the relation

* G. N. Watson, Theory of Bessel Functions, Cambridge, 1922, p. 181, (1).

† Watson, loc. cit., p. 183, (15).

$\ddagger$ Watson, loc. cit., p. 78, (6). 


$$
\begin{aligned}
k^{r}\left(\frac{c_{o}}{\left|\sigma_{h, k}^{(o)}\right| q_{\beta}}\right)^{r}\left\{L_{h, k, \nu}^{(o)}(n)+M_{h, k, \nu}^{(o)}(n)\right\} \\
=\frac{2 \pi\left|\sigma_{h, k}^{(o)}\right| q_{\beta}\left(\nu-\alpha_{\beta}\right)^{(r+1) / 2} c_{o}^{(r-1) / 2}}{k c_{\beta}^{(r+1) / 2}\left(\alpha_{o}+n\right)^{(r+1) / 2}} \\
\cdot I_{r+1}\left(\frac{4 \pi\left|\sigma_{h, k}^{(o)}\right| q_{\beta}\left(\left(\nu-\alpha_{\beta}\right)\left(\alpha_{o}+n\right)\right)^{1 / 2}}{k\left(c_{\beta} c_{o}\right)^{1 / 2}}\right) .
\end{aligned}
$$

Using this result in (5.8) we have the following theorem:

THEOREM 1. Let $F(\tau)$ be a modular form of positive dimension $r$ belonging to the subgroup $\gamma$, and let its expansions (3.11) have only a finite number of terms with negative exponent. Then the coefficients ${a_{n}}^{(0)}$ of (3.12) for which $\alpha_{0}+n>0$ are given by

$$
\begin{aligned}
a_{n}^{(\sigma)}= & \frac{2 \pi c_{o}^{(r-1) / 2}}{\left(\alpha_{\theta}+n\right)^{(r+1) / 2}} \sum_{h, k}^{\infty} \Omega_{h, k}^{(o)} \exp \left\{-2 \pi i n \frac{h}{k}\right\} \\
& \cdot \sum_{\nu=1}^{\mu_{\beta}} a_{-\nu}^{(\beta)} \exp \left\{-\nu G_{h, k}^{(o)} i\right\} \frac{\left|\sigma_{h, k}^{(h)}\right| q_{\beta}\left(\nu-\alpha_{\beta}\right)^{(r+1) / 2}}{k c_{\beta}^{(r+1) / 2}} \\
& \cdot I_{r+1}\left(\frac{4 \pi\left|\sigma_{h, k}^{(o)}\right| q_{\beta}\left(\left(\nu-\alpha_{\beta}\right)\left(\alpha_{o}+n\right)\right)^{1 / 2}}{k\left(c_{\beta} c_{o}\right)^{1 / 2}}\right)
\end{aligned}
$$

where $\beta=\beta(h, k, g)$ is defined in $\$ 4 ; c_{\beta}$ and $c_{0}$ are determined by $(3.11) ; \alpha_{\beta}$ and $\alpha_{0}$ by (3.12); $\Omega_{n, \mathrm{k}}^{(0)}$ is defined in (4.32); and $G_{h, \mathrm{k}}^{(0)}$ in (4.34). The $F^{*}(\tau)$ of (1.32) are then given by (3.23).

7. Application to a particular subgroup. Many of the constants in (6.3) depend merely on the subgroup $\gamma$ and are otherwise independent of the choice of $F(\tau)$. For this reason we can simplify the expression if we choose some particular subgroup $\gamma$. As an example we consider the subgroup consisting of all transformations

$$
\tau^{\prime}=\frac{a \tau+b}{c \tau+d}, \quad a \equiv d \equiv 1, c \equiv 0(\bmod 2) .
$$

For the region $R$ we may take that part of the plane which is above the two circles $|\tau-1 / 4|=1 / 4$ and $|\tau-3 / 4|=1 / 4$ and which is within the circle $|\tau-1 / 2|=1 / 2$. Then $R$ has the three parabolic points $0,1 / 2$, and 1 , but 0 and 1 are congruent under $\gamma$; so we have $s=2$ and take $P_{1}=0, P_{2}=1 / 2$. Then we have

$$
p_{1}=0, \quad q_{1}=1, \quad p_{2}=1, \quad q_{2}=2 .
$$

Corresponding to the transformations (3.11) we have the two transformations 


$$
\tau^{\prime}=\frac{\tau}{2 \tau+1}, \quad \tau^{\prime}=\frac{3 \tau-1}{4 \tau-1}
$$

which may be written in the form

$$
\frac{1}{\tau^{\prime}}=\frac{1}{\tau}+2, \quad \frac{1}{\tau^{\prime}-1 / 2}=\frac{1}{\tau-1 / 2}+4 ;
$$

so we have $c_{1}=2$ and $c_{2}=4$. The expansions (3.12) can now be written as
(7.21) $\quad F(\tau)=(-i \tau)^{r} x^{\alpha_{1}} \sum_{m=-\mu_{1}}^{\infty} a_{m}^{(1)} x^{m}$,$$
x=\exp \left\{-\frac{\pi i}{\tau}\right\}
$$$$
F(\tau)=(-i(\tau-1 / 2))^{r} x^{\alpha_{2}} \sum_{m=-\mu_{2}}^{\infty} a_{m}^{(2)} x^{m}, \quad x=\exp \left\{-\frac{\pi i}{2 \tau-1}\right\} .
$$

In order to determine $\beta(h, k, g)$ we find transformations (7.1) which transform $P_{0}-k / c_{0} h$ into $P_{\beta}$. We define $h^{\prime}, h^{\prime \prime}, h^{\prime \prime \prime}$, and $h^{\text {iv }}$ as any solutions of

$$
\begin{aligned}
k h^{\prime} & \equiv 1 \quad(\bmod 2 h), & h^{\prime}>0, \text { for } k & \equiv 1(\bmod 2) ; \\
k h^{\prime \prime} & \equiv-1(\bmod h), & h^{\prime \prime} & >0, \text { for } k \equiv 0(\bmod 2) ; \\
(k-2 h) h^{\prime \prime \prime} & \equiv 1 \quad(\bmod 4 h), & h^{\prime \prime \prime}>0, \text { for } k & \equiv 1(\bmod 2) ; \\
\left(\frac{k}{2}-h\right) h^{\mathrm{iv}} & \equiv 1 \quad(\bmod 2 h), & h^{\mathrm{iv}}>0, \text { for } k & \equiv 0(\bmod 4) ;
\end{aligned}
$$

and may take for $a, b, c$, and $d$ the values given in the following tables.

\begin{tabular}{|c|c|c|c|c|}
\hline$g$ & $k$ & $a$ & $b$ & $c$ \\
\hline 1 & $\equiv 1(\bmod 2)$ & $h^{\prime}$ & $\frac{h^{\prime} k-1}{2 h}$ & $2 h^{\prime}+2 h$ \\
\hline 1 & $\equiv 0(\bmod 2)$ & $h$ & $\frac{k}{2}$ & $2 h^{\prime \prime}$ \\
\hline 2 & $\equiv 1(\bmod 2)$ & $h^{\prime \prime \prime}$ & $\frac{(k-2 h) h^{\prime \prime \prime}-1}{4 h}$ & $2 h^{\prime \prime \prime}+4 h$ \\
\hline 2 & $\equiv 0(\bmod 4)$ & $h^{\mathrm{iv}}$ & $\frac{\left(\frac{k}{2}-h\right) h^{\mathrm{iv}}-1}{2 h}$ & $2 h^{\mathrm{iv}}+2 h$ \\
\hline 2 & $\equiv 2(\bmod 4)$ & $h$ & $\frac{k-2 h}{4}$ & $4 h^{\prime \prime}$ \\
\hline
\end{tabular}




\begin{tabular}{|c|r|r|r|r|}
\hline$d$ & $\beta$ & $\sigma_{h, k}^{(o)}$ & $\delta_{h, k}^{(o)}$ & $G_{h, k}^{(o)}$ \\
\hline$\frac{h^{\prime} k-1}{h}+k$ & 2 & -1 & 1 & $\left(\frac{h^{\prime} k-1}{k h}+1\right) \pi$ \\
\hline$\frac{1+h^{\prime \prime} k}{h}$ & 1 & 2 & -1 & $-\left(\frac{2+2 h^{\prime \prime} k}{k h}\right) \pi$ \\
\hline$\frac{(k-2 h) h^{\prime \prime \prime}-1}{2 h}-2 h+k$ & 2 & -1 & 1 & $\left(\frac{h^{\prime \prime \prime} k-1}{2 k h}+1\right) \pi$ \\
\hline$\frac{\left(\frac{k}{2}-h\right) h^{\mathrm{iv}}-1}{h}+\frac{k}{2}-h$ & 2 & -2 & 1 & $\left(\frac{h^{\mathrm{iv}} k-2}{k h}+1\right) \pi$ \\
\hline$\frac{k h^{\prime \prime}+1}{h}-2 h^{\prime \prime}$ & 1 & 4 & -1 & $-\left(\frac{4 h^{\prime \prime} k+4}{k h}\right) \pi$ \\
\hline
\end{tabular}

The values of $\sigma_{h, k}^{(o)}$ were found by means of (4.13) and $G_{h, \mathbf{k}}^{(0)}$ from (4.34).

The series (6.3) can be shown to be absolutely convergent; so we may rearrange the terms. Doing this and making use of (4.32) and the tables, we get

$$
\begin{aligned}
a_{n}^{(1)}= & 2 \pi \sum_{\nu=1}^{\mu_{1}} a_{-\nu}^{(1)} \sum_{\substack { k \equiv 0 \\
\begin{subarray}{c}{(\bmod 2) \\
k>0{ k \equiv 0 \\
\begin{subarray} { c } { ( \operatorname { m o d } 2 ) \\
k > 0 } }\end{subarray}} \frac{1}{k} A_{k, \nu}^{(1)}(n) \\
& \cdot\left(\frac{\nu-\alpha_{1}}{n+\alpha_{1}}\right)^{(r+1) / 2} I_{r+1}\left(\frac{4 \pi\left(\left(\nu-\alpha_{1}\right)\left(n+\alpha_{1}\right)\right)^{1 / 2}}{k}\right) \\
& +\frac{\pi}{2^{(r-1) / 2}} \sum_{\nu=1}^{\mu_{2}} a_{-\nu}^{(2)} \sum_{k \equiv 1 \text { (mod2) }} \frac{1}{k} A_{k, \nu}^{(1)}(n) \\
& \cdot\left(\frac{\nu-\alpha_{2}}{n+\alpha_{1}}\right)^{(r+1) / 2} I_{r+1}\left(\frac{4 \pi\left(\left(\nu-\alpha_{2}\right)\left(n+\alpha_{1}\right)\right)^{1 / 2}}{k 2^{1 / 2}}\right),
\end{aligned}
$$

where

$$
\begin{aligned}
& A_{k, \nu}^{(1)}(n)= \sum_{\substack{0 \leqq h<k \\
(h, k)=1}} \epsilon\left(h, \frac{k}{2}, 2 h^{\prime \prime}, \frac{1+h^{\prime \prime} k}{h}\right)^{-1} \exp \{\pi i r\} \\
& \cdot \exp \left\{-\frac{2 \pi i}{k} \alpha_{1} h-\left(\alpha_{1}-\nu\right)\left(\frac{2 h^{\prime \prime} k+2}{k h}\right) \pi i-2 \pi i n \frac{h}{k}\right\}, \\
& \text { if } k \equiv 0(\bmod 2),
\end{aligned}
$$




$$
\begin{aligned}
A_{k, \nu}^{(1)}(n)= & \sum_{\substack{0 \leqq h<k \\
(h, k)=1}} \epsilon\left(h^{\prime}, \frac{h^{\prime} k-1}{2}, 2 h^{\prime}+2 h, \frac{h^{\prime} k-1}{h}+k\right)^{-1} \\
& \cdot \exp \left\{-\frac{2 \pi i}{k} \alpha_{1} h+\left(\alpha_{2}-\nu\right)\left(\frac{h^{\prime} k-1}{k h}+1\right) \pi i-2 \pi i n \frac{h}{k}\right\},
\end{aligned}
$$

if $k \equiv 1(\bmod 2)$,

if $g=1$ for all $n$ such that $\alpha_{1}+n>0$. Similarly, if $g=2$ and $\alpha_{2}+n>0$, we have

$$
\begin{aligned}
& a_{n}^{(2)}=2^{(r+3) / 2} \pi \sum_{\nu=1}^{\mu_{1}} a_{-v}^{(1)} \sum_{k \equiv 2(\bmod 4)} \frac{1}{k} A_{k, \nu}^{(2)}(n)\left(\frac{\nu-\alpha_{1}}{n+\alpha_{2}}\right)^{(r+1) / 2} \\
& \cdot I_{r+1}\left(\frac{8 \pi\left(\left(\nu-\alpha_{1}\right)\left(n+\alpha_{2}\right)\right)^{1 / 2}}{k 2^{1 / 2}}\right)+2 \pi \sum_{\nu=1}^{\mu_{2}} a_{-\nu}^{(2)} \sum_{\substack{k \equiv 0 \text { (mod4) } \\
k>0}} \frac{1}{k} A_{k, \nu}^{(2)}(n) \\
& \cdot\left(\frac{\nu-\alpha_{2}}{n+\alpha_{2}}\right)^{(r+1) / 2} I_{r+1}\left(\frac{4 \pi\left(\left(\nu-\alpha_{2}\right)\left(n+\alpha_{2}\right)\right)^{1 / 2}}{k}\right) \\
& +\pi \sum_{\nu=1}^{\mu_{2}} a_{-\nu}^{(2)} \sum_{k \equiv 1} \frac{1}{k>0} A_{k>0}^{(2)}(n) \\
& \cdot\left(\frac{\nu-\alpha_{2}}{n+\alpha_{2}}\right)^{(r+1) / 2} I_{r+1}\left(\frac{2 \pi\left(\left(\nu-\alpha_{2}\right)\left(n+\alpha_{2}\right)\right)^{1 / 2}}{k}\right),
\end{aligned}
$$

where

$$
\begin{aligned}
& A_{k, \nu}^{(2)}(n) \\
& =\sum_{\substack{0 \leqq h<k \\
(h, k)=1}} \epsilon\left(h, \frac{k-2 h}{4}, 4 h^{\prime \prime}, \frac{k h^{\prime \prime}+1}{h}-2 h^{\prime \prime}\right)^{-1} \exp \{\pi i r\} \\
& \quad \cdot \exp \left\{-\frac{2 \pi i}{k} \alpha_{2} h-\left(\alpha_{1}-\nu\right)\left(\frac{2 h^{\prime \prime} k+2}{k h}\right) \pi i-2 \pi i n \frac{h}{k}\right\},
\end{aligned}
$$

if $k \equiv 2(\bmod 4)$,

$A_{k, v}^{(2)}(n)$

(7.46)

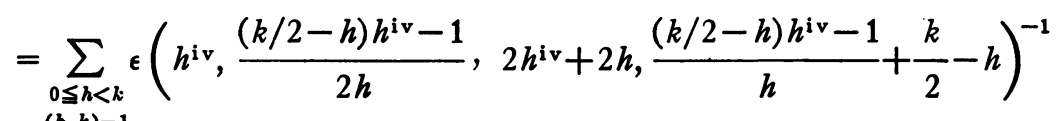

$$
\cdot \exp \left\{-\frac{2 \pi i}{k} \alpha_{2} h+\left(\alpha_{2}-\nu\right)\left(\frac{h^{\mathrm{iv}} k-2}{k h}+1\right) \pi i-2 \pi i n \frac{h}{k}\right\},
$$

$$
\text { if } k \equiv 0(\bmod 4) \text {, }
$$




$$
\begin{aligned}
& A_{k, \nu}^{(2)}(n) \\
&= \sum_{\substack{0 \leq h<k \\
(h, k)=1}} \epsilon\left(h^{\prime \prime \prime}, \frac{(k-2 h) h^{\prime \prime \prime}-1}{4 h}, 2 h^{\prime \prime \prime}+4 h, \frac{(k-2 h) h^{\prime \prime \prime}-1}{2 h}-2 h+k\right)^{-1} \\
& \exp \left\{-\frac{2 \pi i}{k} \alpha_{2} h+\left(\alpha_{2}-\nu\right)\left(\frac{h^{\prime \prime \prime} k-1}{2 k h}+1\right) \pi i-2 \pi i n \frac{h}{k}\right\}, \\
& \text { if } k \equiv 1(\bmod 2) .
\end{aligned}
$$

Equation (7.44) can be given a more symmetric form by changing the indices of summation. In the first sum we set $k=2 l$ in the second $k=l$, and in the third $k=l / 2$. Then we find

$$
\begin{aligned}
& a_{n}^{(2)}=2^{(r+1) / 2} \pi \sum_{n=1}^{\mu_{1}} a_{-\nu}^{(1)} \sum_{\substack{l=1(\bmod 2) \\
l>0}} \frac{1}{l} A_{2 l, \nu}^{(2)}(n) \\
& \cdot\left(\frac{\nu-\alpha_{1}}{n+\alpha_{2}}\right)^{(r+1) / 2} I_{r+1}\left(\frac{4 \pi\left(\left(\nu-\alpha_{1}\right)\left(n+\alpha_{2}\right)\right)^{1 / 2}}{l 2^{1 / 2}}\right) \\
& +2 \pi \sum_{n=1}^{\mu_{2}} a_{-v}^{(2)} \sum_{\substack{l=0(\bmod 4) \\
l>0}} \frac{1}{l} A_{l, \nu}^{(2)}(n) \text {. } \\
& \cdot\left(\frac{\nu-\alpha_{2}}{n+\alpha_{2}}\right)^{(r+1) / 2} I_{r+1}\left(\frac{4 \pi\left(\left(\nu-\alpha_{2}\right)\left(n+\alpha_{2}\right)\right)^{1 / 2}}{l}\right) \\
& +2 \pi \sum_{v=1}^{\mu_{2}} a_{-v}^{(2)} \sum_{\substack{l \equiv 2(\bmod 4) \\
l>0}} \frac{1}{l} A_{l / 2, \nu}^{(2)}(n) \\
& \cdot\left(\frac{\nu-\alpha_{2}}{n+\alpha_{2}}\right)^{(r+1) / 2} I_{r+1}\left(\frac{4 \pi\left(\left(\nu-\alpha_{2}\right)\left(n+\alpha_{2}\right)\right)^{1 / 2}}{l}\right) \\
& =2^{(r+1) / 2} \pi \sum_{v=1}^{\mu_{1}} a_{-v}^{(1)} \sum_{\substack{l \equiv 1(\bmod 2) \\
l>0}} \frac{1}{l} A_{l^{\prime}, v}^{(2)}(n) \\
& \cdot\left(\frac{\nu-\alpha_{1}}{n+\alpha_{2}}\right)^{(r+1) / 2} I_{r+1}\left(\frac{4 \pi\left(\left(\nu-\alpha_{1}\right)\left(n+\alpha_{2}\right)\right)^{1 / 2}}{l 2^{1 / 2}}\right) \\
& +2 \pi \sum_{v=1}^{\mu_{2}} a_{-\nu}^{(2)} \sum_{\substack{l \equiv 0(\bmod 2) \\
l>0}} \frac{1}{l} A_{l^{\prime}, \nu}^{(2)}(n) \\
& \cdot\left(\frac{\nu-\alpha_{2}}{n+\alpha_{2}}\right)^{(r+1) / 2} I_{r+1}\left(\frac{4 \pi\left(\left(\nu-\alpha_{2}\right)\left(n+\alpha_{2}\right)\right)^{1 / 2}}{l}\right)
\end{aligned}
$$


where

(7.49) $l^{\prime}=2 l$ if $l \equiv 1(\bmod 2), l^{\prime}=l$ if $l \equiv 0(\bmod 4), l^{\prime}=l / 2$ if $l \equiv 2(\bmod 4)$.

We now find the functions $F^{*}(\tau)$ of (1.32). If $C$ is odd and $D$ even, we take

$$
\tau^{\prime}=\frac{ \pm B \tau \mp A}{ \pm D \tau \mp C}
$$

for the transformation (3.21) where we take the upper or lower signs to make $\pm D \geqq 0$. This transformation takes $P_{1}=0$ into $A / C$ and it belongs to $\gamma$. From (3.22) we find $\kappa=\mp 1$ and from (3.23) and (3.24) we have

$$
\begin{aligned}
F^{*}(\tau) & =e^{\pi i \alpha_{1} \tau} f_{1}\left(e^{\pi i \tau}\right)=e^{\pi i \alpha_{1} \tau} \sum_{m=-\mu_{1}}^{\infty} a_{m}(1) e^{\pi i m \tau} \\
\epsilon^{*} & =\epsilon( \pm B, \mp A, \pm D, \mp C) e^{\mp \pi i r / 2}
\end{aligned}
$$

Similarly if $C$ and $D$ are both odd, we use the transformation

$$
\tau^{\prime}=\frac{ \pm(B+A) \tau \mp A}{ \pm(D+C) \tau \mp C}, \quad \quad \pm(D+C) \geqq 0,
$$

and obtain

$$
\begin{aligned}
F^{*}(\tau) & =e^{\pi i \alpha_{1} \tau} f_{1}\left(e^{-\pi i} e^{\pi i \tau}\right)=e^{\pi i \alpha_{1} \tau} \sum_{m=-\mu_{1}}^{\infty}(-1)^{m} a_{m}{ }^{(1)} e^{\pi i m \tau} \\
\epsilon^{*} & =\epsilon( \pm(B+A), \mp A, \pm(D+C), \mp C) e^{\mp i r / 2} e^{-\pi i \alpha_{1}}
\end{aligned}
$$

Finally if $C$ is even, we use the transformation

$$
\tau^{\prime}=\frac{ \pm(A+2 B) \tau \mp(A+B)}{ \pm(C+2 D) \tau \mp(C+D)}, \quad \pm(C+2 D) \geqq 0,
$$

which takes $P_{2}=1 / 2$ into $A / C$ and find

$$
\begin{aligned}
F^{*}(\tau) & =2^{-\tau} e^{2 \pi i \alpha_{2} \tau} f_{2}\left(e^{-\pi i} e^{2 \pi i \tau}\right)=2^{-\tau} e^{2 \pi i \alpha_{2} \tau} \sum_{m=-\mu_{3}}^{\infty}(-1)^{m} a_{m}(2) e^{2 \pi i m \tau}, \\
\epsilon^{*} & =\epsilon( \pm(A+2 B), \mp(A+B), \pm(C+2 D), \mp(C+D)) e^{\mp \pi i r / 2} e^{-\pi i \alpha_{2}}
\end{aligned}
$$

Making use of (1.32) we summarize the results of this section in the following theorem:

THEOREM 2. If $F(\tau)$ is a modular form of positive dimension belonging to the subgroup (7.1) and if its expansions (7.21) and (7.22) have only a finite number of terms with negative exponent, then the values of $a_{n}^{(0)}$ for $\alpha_{o}+n>0$, $(g=1,2)$, are determined as functions of $a_{n}^{(0)}$ with $n<0$ by (7.41) and (7.48).

Also we have 


$$
F\left(\frac{A \tau+B}{C \tau+D}\right)=\epsilon^{*}(-i(C \tau+D))^{-\tau} F^{*}(\tau)
$$

for any modular transformation $\tau^{\prime}=(A \tau+B) /(C \tau+D)$ where $\epsilon^{*}$ and $F^{*}(\tau)$ are given by (7.51) for $C$ odd, $D$ even; by (7.52) for $C$ odd, $D$ odd; and by (7.53) for $C$ even.

8. An example. As a particular example we consider the function

$$
F(\tau)=\left(\vartheta_{2}(0 \mid \tau)\right)^{-1}
$$

which belongs to the subgroup discussed in $\$ 7$. From the theory of the thetafunctions we take only the following results: $\dagger$

$$
\vartheta_{2}\left(0 \mid \frac{a \tau+b}{c \tau+d}\right)=\epsilon^{\prime}( \pm c \tau \pm d)^{1 / 2} \vartheta_{2}(0 \mid \tau)
$$

if $a$ and $d$ are odd and $c$ is even where we take the upper or lower sign to make $\pm d>0$ and where

$$
\epsilon^{\prime}=\left(\frac{ \pm c}{ \pm d}\right) \exp \left\{\frac{\pi i}{4}(b d \pm 5 d-5)\right\}
$$

$$
\begin{aligned}
\vartheta_{2}\left(0 \mid-\frac{1}{\tau}\right) & =(-i \tau)^{1 / 2} \vartheta_{4}(0 \mid \tau) ; \\
\vartheta_{2}\left(0 \mid \frac{-1}{\tau+1}\right) & =(-i(\tau+1))^{1 / 2} \vartheta_{3}(0 \mid \tau) ; \\
\vartheta_{2}(0 \mid \tau) & =2 e^{\pi i \tau / 4}+2 e^{9 \pi i \tau / 4}+\cdots ; \\
\vartheta_{4}(0 \mid \tau) & =1-2 e^{\pi i \tau}+\cdots .
\end{aligned}
$$

From (8.11), (8.12), and (8.13) we see that $F(\tau)$ satisfies a transformation equation of the type (1.11) with $r=1 / 2$ and

$$
\epsilon=\epsilon(a, b, c, d)=\left(\frac{ \pm c}{ \pm d}\right) \exp \left\{-\frac{\pi i}{4}(b d \pm 5 d-5 \pm 1)\right\}, \pm d>0
$$

for all transformations of the subgroup. By (8.16) we have

$$
F(\tau)=\left(2 e^{\pi i \tau / 4}+2 e^{9 \pi i \tau / 4}+\cdots\right)^{-1}=e^{i \pi i \tau / 4}\left(\frac{1}{2} e^{-2 \pi i \tau}-\frac{1}{2}+\cdots\right)
$$

and by (8.14) and (8.17) we have

$$
F(-1 / \tau)=(-i \tau)^{-1 / 2}\left(1+2 e^{\pi i \tau}+\cdots\right) .
$$

On the other hand, (7.6) and (7.53) with $A=D=1, B=C=0$ yield 


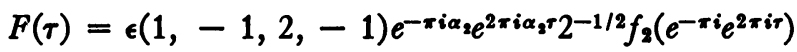

$$
\begin{aligned}
& =2^{-1 / 2} e^{-\pi i \alpha_{2}} e^{2 \pi i \alpha_{2} \tau} \sum_{m=-\mu_{2}}^{\infty}(-1)^{m} a_{m}{ }^{(2)} e^{2 \pi i m \tau},
\end{aligned}
$$

and (7.6) and (7.51) with $A=D=0, B=-1, C=1$ give

$$
F(-1 / \tau)=(-i \tau)^{-1 / 2} e^{\pi i \alpha_{1} \tau} \sum_{m=-\mu_{1}}^{\infty} a_{m}{ }^{(1)} e^{\pi i m r} .
$$

Comparing (8.22) with (8.24) and (8.23) with (8.25), we find

(8.26) $\quad \alpha_{1}=0, \quad \alpha_{2}=7 / 8, \quad \mu_{1}=0, \quad \mu_{2}=1, \quad a_{-1}^{(2)}=2^{-1 / 2} e^{-\pi i / 8}, \quad a_{0}^{(1)}=1$.

Using these values we find that equations (7.41) and (7.43) now reduce to

$$
a_{n}^{(1)}=2^{-6 / 2} \pi e^{-\pi i / 8} \sum_{\substack{k=1(\bmod 2) \\ k>0}} \frac{1}{k} A_{k, 1}^{(1)}(n) \frac{1}{n^{3 / 4}} I_{3 / 2}\left(\frac{\pi n^{1 / 2}}{k}\right), \quad n \geqq 1,
$$

where

$$
\begin{aligned}
A_{k, \mathrm{I}}^{(1)}(n)= & \sum_{\substack{0 \leq h<k \\
(h, k)-1}} \epsilon\left(h^{\prime}, \frac{h^{\prime} k-1}{2 h}, 2 h^{\prime}+2 h, \frac{h^{\prime} k-1}{h}+k\right)^{-1} \\
& \cdot \exp \left\{-\frac{\pi i}{8}\left(\frac{h^{\prime} k-1}{k h}+1\right)-2 \pi i n \frac{h}{k}\right\}, k \equiv 1(\bmod 2),
\end{aligned}
$$

with $h^{\prime}$ defined by (7.31) and the $\epsilon$ of (8.21). Similarly (7.48), (7.46), and (7.47) reduce to

$$
\begin{aligned}
a_{n}^{(2)}= & 2^{-7 / 4} \pi e^{-\pi i / 8} \sum_{\substack{l \equiv 0(\bmod 2) \\
>0}} \frac{1}{l} A_{l^{\prime}, 1}^{(2)}(n) \frac{1}{(n+7 / 8)^{3 / 4}} & \\
& \cdot I_{3 / 2}\left(\frac{2 \pi(n+7 / 8)^{1 / 2}}{l 2^{1 / 2}}\right), & n \geqq 0,
\end{aligned}
$$

where $l^{\prime}$ is given by (7.49) and

$$
\begin{aligned}
& A_{k, 1}^{(2)}(n) \\
& =\sum_{\substack{0 \leq h<k \\
(h, k)=1}} \epsilon\left(h^{\mathrm{iv}} \frac{(k / 2-h) h^{\mathrm{iv}}-1}{2 h}, 2 h^{\mathrm{iv}}+2 h, \frac{(k / 2-h) h^{\mathrm{iv}}-1}{h}+\frac{k}{2}-h\right)^{-1} \\
& \quad \cdot \exp \left\{-\frac{7 \pi i}{4} \frac{h}{k}-\frac{\pi i}{8}\left(\frac{h^{\mathrm{iv}} k-2}{k h}+1\right)-2 \pi i n \frac{h}{k}\right\}, \text { if } k \equiv 0(\bmod 4),
\end{aligned}
$$




$$
\begin{aligned}
& A_{k, 1}^{(2)}(n) \\
& =\sum_{\substack{0 \leqq h<k \\
(h, k)=1}} \epsilon\left(h^{\prime \prime \prime}, \frac{(k-2 h) h^{\prime \prime \prime}-1}{4 h}, 2 h^{\prime \prime \prime}+4 h, \frac{(k-2 h) h^{\prime \prime \prime}-1}{2 h}-2 h+k\right)^{-1} \\
& \quad \cdot \exp \left\{-\frac{7 \pi i}{4} \frac{h}{k}-\frac{\pi i}{8}\left(\frac{h^{\prime \prime \prime} k-1}{2 k h}+1\right)-2 \pi i n \frac{h}{k}\right\}, \text { if } k \equiv 1(\bmod 2),
\end{aligned}
$$

with $h^{\prime \prime \prime}$ and $h^{\text {iv }}$ defined by (7.33) and (7.34) and the $\epsilon$ of (8.21).

The Bessel functions which occur in (8.31) and (8.33) are of order half an odd integer and hence may be expressed in terms of elementary functions. Doing this we find that these equations may be written in the form

$$
a_{n}^{(1)}=\frac{1}{2 \pi} e^{-\pi i / 8} \sum_{\substack{k \equiv 1(\bmod 2) \\ k>0}} k^{1 / 2} A_{k, 1}^{(1)}(n) \frac{d}{d n}\left(\frac{\sinh \frac{\pi}{k} n^{1 / 2}}{n^{1 / 2}}\right)
$$

and

$$
a_{n}^{(2)}=\frac{1}{2 \pi} e^{-\pi i / 8} \sum_{\substack{l \equiv 0(\bmod 2) \\ l>0}} l^{1 / 2} A_{l^{\prime}, 1}^{(2)}(n) \frac{d}{d n}\left(\frac{\sinh \frac{\pi}{l}(2(n+7 / 8))^{1 / 2}}{(n+7 / 8)^{1 / 2}}\right) .
$$

The expansion of $F((A \tau+B) /(C \tau+D))$, for $\tau^{\prime}=(A \tau+B) /(C \tau+D)$ any modular transformation, can now be obtained from (7.6). However we shall consider only three particular cases from which we shall get expansions for $\vartheta_{2}(0 \mid \tau)^{-1}, \vartheta_{3}(0 \mid \tau)^{-1}$, and $\vartheta_{4}(0 \mid \tau)^{-1}$. Two of these are to be found from (8.24) and (8.25). We insert the values (8.26) and have

$$
F(\tau)=2^{-1 / 2} e^{-7 \pi i / 8} e^{7 \pi i \tau / 4} \sum_{m=-1}^{\infty}(-1)^{m} a_{m}{ }^{(2)} e^{2 \pi i m \tau}
$$

and

$$
F(-1 / \tau)=(-i \tau)^{-1 / 2} \sum_{m=0}^{\infty} a_{m}^{(1)} e^{\pi i m \tau}
$$

The third expansion is obtained from (7.6) and (7.52) by taking $A=0$, $B=-1, C=D=1$, and the values (8.26). We then find

$$
F\left(\frac{-1}{\tau+1}\right)=(-i(t+1))^{-1 / 2} \sum_{m=0}^{\infty}(-1)^{m} a_{m}^{(1)} e^{\pi i m r} .
$$


A comparison of (8.11) with (8.41), (8.15) with (8.43), and (8.14) with (8.42) then yields the desired expansions

$$
\vartheta_{2}(0 \mid \tau)^{-1}=2^{-1 / 2} e^{-7 \pi i / 8} e^{7 \pi i \tau / 8} \sum_{m=-1}^{\infty}(-1)^{m} a_{m}^{(2)} e^{2 \pi i m \tau},
$$

$$
\vartheta_{8}(0 \mid \tau)^{-1}=\sum_{m=0}^{\infty}(-1)^{m} a_{m}^{(1)} e^{\pi i m \tau},
$$

$$
\vartheta_{4}(0 \mid \tau)^{-1}=\sum_{m=0}^{\infty} a_{m}^{(1)} e^{\pi i m \tau}
$$

University of PenNsylvania,

Philadelphia, Pa. 